\title{
Adjoint Algorithms for the Optimization of 3d Turbulent Configurations
}

\author{
Richard P. Dwight ${ }^{1}$ and Joël Brezillon ${ }^{1}$ \\ German Aerospace Center (DLR), Lilienthalplatz, 7, Braunschweig, D-38108 \\ Germany. \\ richard.dwight@dlr.de, joel.brezillon@dlr.de
}

\begin{abstract}
Summary
The solution of the discrete adjoint equations for an unstructured finite volume compressible Navier-Stokes solver is discussed. In previous work fixedpoint iterations taken from the non-linear method - suitably adjointed - were applied to the adjoint problem. Here it is seen that there are often situations in which these iterations can not be expected to converge. To address this the Recursive Projection Method is developed as a stabilizer, and then used to perform an eigenmode analysis of attached and separated flow on a single geometry, allowing identification of flow regions that were unstable under the basic iteration. Finally an adjoint based optimization with 96 design variables is performed on a wing-body configuration. The initial flow has large regions of separation, which are significantly diminished in the optimized configuration.
\end{abstract}

\section{Introduction}

The adjoint equations are enjoying increasing importance in the field of computational aerodynamics, as emphasis shifts from the modelling of physical phenomena to their control and optimization. Adjoint algorithms are necessary to evaluate cost-function sensitivities with respect to a large number of design variables with an effort only weakly dependant on their number.

But solving the adjoint equations is a problem at least as hard as solving the original flow equations, and often significantly harder. One possibility is to apply the same iterative techniques used for the non-linear problem, suitably adjointed, to the adjoint problem. This technique guarantees adjoint convergence if the non-linear problem converges asymptotically [1] - but this is often not the case. For this reason the Recursive Projection Method (RPM) is studied as a stabilizer, Section 2. The approximate eigenmodes that are calculated within RPM are useful for identifying the cause of divergence of the original iteration, and in our particular cases the cause is found to be flow separation, see Section 3. The algorithms described are finally applied to optimization of a $3 \mathrm{~d}$ turbulent wing-body configuration. 
We forego a detailed description of the compressible Navier-Stokes equations and their unstructured finite volume discretization in the DLR TAUCode, referring the interested reader to $[2,3]$, and note only that the method uses the Jameson-Schmitt-Turkel (JST) flux with scalar artificial dissipation and the one-equation Spalart-Allmaras turbulence model with Edwards modification.

\section{The Recursive Projection Method}

Despite the guarantees regarding convergence provided by the theory of adjointed fixed-point iterations (FPIs) [1] there are regularly situations in which it is possible to obtain a reasonably converged solution of the non-linear equations, but not of the corresponding adjoint equations. This can occur for three reasons: either a) the non-linear solution is not sufficiently converged, or b) there is a discrepancy between the linear and non-linear problem due to some approximation of the Jacobian, or c) the FPI applied to the non-linear problem does not converge asymptotically itself.

In an effort to understand and mitigate these phenomena, we consider the Recursive Projection Method (RPM), originally developed by Schroff and Keller in 1993 as a means of stabilization of unstable procedures [4]. Since then it has been applied in aerodynamics for convergence acceleration [5], and stabilization of linear frequency domain solvers [6].

Let the (linear) adjoint system be written $A x=b$. The idea of RPM is to regard the transient solution of the linear problem as a sum of eigenvectors of the relaxation operator $\Phi=\left(I-M^{-1} A\right)$ where $M$ is some iteration operator, e.g. LU-SGS with multigrid. The application of $\Phi$ to an approximate solution then corresponds to a product of each eigenvector with its corresponding eigenvalue. Divergence of the iteration implies that there is at least one eigenvalue of $\Phi$ with modulus greater than unity. Assuming that the number of such eigenvalues is small, and that the space spanned by their eigenvectors is known, call it $\mathcal{P}$, then it must be possible to solve the projection of the problem onto this low dimensional subspace using some expensive but stable method, while solving the projection onto the complimentary subspace $\mathcal{Q}$ using the original FPI iteration, which is known to be stable there.

Newton-Raphson is typically used on $\mathcal{P}$. The space of dominant eigenvectors is determined as the calculation progresses, by applying the principle that the difference between successive applications of the FPI on $Q$ form a power iteration on the dominant eigenvalues of $\Phi$ restricted to $Q$.

In more detail: consider a relaxation operator written

$$
N(x)=\left(I-M^{-1} A\right) x+M^{-1} b .
$$

Let $V$ be an orthonormal basis of $\mathcal{P}$, then orthogonal projection operators onto $\mathcal{P}$ and $\mathcal{Q}$ may be written respectively $P=V V^{T}$ and $Q=I-V V^{T}$. 
Further define $x_{P}=P x, x_{Q}=Q x$. Then the RPM iteration may be written

$$
\begin{aligned}
& x_{Q}^{n+1}=Q N\left(x^{n}\right), \\
& x_{P}^{n+1}=x_{P}^{n}+(I-P \Phi P)^{-1}\left[P N\left(x^{n}\right)-x_{P}^{n}\right],
\end{aligned}
$$

where

$$
x^{n+1}=x_{P}^{n+1}+x_{Q}^{n+1} .
$$

The derivative term in the Newton iteration (3) may be written $V(I-$ $H)^{-1} V^{T}$, where $H$ is a square matrix of size dimension of $\mathcal{P}$, whose inversion is cheap if $\mathcal{P}$ is of low dimension.

To determine the basis itself consider the sequence

$$
K=\left[\Delta x_{Q}^{n}, \Delta x_{Q}^{n-1}, \ldots, \Delta x_{Q}^{n-k+1}\right],
$$

where $\Delta x_{Q}^{n}=x_{Q}^{n+1}-x_{Q}^{n}$ are readily available from the iteration on Q. Using the linearity of $\Phi$ it is easy to show that $\Delta x_{Q}^{n}=Q \Phi Q \Delta x^{n-1}$, so $K$ is a Krylov space for $Q \Phi Q$. Asymptotically this subspace will tend to contain the dominant $k$ eigenvectors of $Q \Phi Q$, as they are the components of the solution most amplified by repeated application of the operator. Via QR factorization an orthogonal basis for $K$ is obtained, and these vectors are added to $V$ when the power iteration becomes sufficiently converged. For more information see [1].

\section{Influence of Separation on Linear Convergence}

To demonstrate the effect unsteady flow phenomena can have on the convergence of the adjoint problem, and to evaluate RPM, we consider two cases: the RAE 2822 aerofoil Case 9 and Case 10 [7]. The only differences between these two cases are the Mach and Reynolds numbers, but Case 9 is fully attached and Case 10 has a large region of shock induced separation. Convergence histories of the flow solutions are shown in Figure 1 where LU-SGS with multigrid with exactly the same settings is used in both cases. The attached case eventually reaches a region of asymptotic convergence, but Case 10 enters a limit cycle after about 1000 iterations and converges no further. However, the lift and drag are well converged, and an engineer might reasonably be satisfied with these values. In fact the parameters of the FPI were specially chosen such that this situation would occur, in a attempt to model a circumstance which is common for complex geometries. The exact cause of the limit cycle is generally difficult to determine with any certainly.

The lack of non-linear asymptotic convergence means that one sufficient condition for convergence of the linear problem has not been met. Figure 1 shows the linear convergence (without RPM), and as expected, the adjoint of Case 9 converges and that of Case 10 diverges. The engineer who then wishes to optimize Case 10 (perhaps to remove the separation) with an adjoint-based 
method can not, even though she can reliably obtain force coefficients. This is clearly an undesirable situation.

Applying RPM then speeds up the convergence of Case 9 and brings Case 10 to convergence, see Figure 1, consistent with the framework developed in the previous section. The discontinuities in the convergence are the iterations at which the basis is extended. As a side effect of RPM the dominant eigenvalues and eigenvectors of $\Phi$ (the LU-SGS smoothed multigrid iteration applied to each case) are approximated, Figure 2. As must be true - given the behaviour of the linear convergence - all eigenvalues of Case 9 lie within the unit circle, and therefore all modes converge. Four eigenvalues of Case 10 lie outside, and the eigenmodes related to these eigenvalues are amplified at each iteration of the scheme. Effectively the diverging components of the problem have been isolated.

Plotting the eigenvectors themselves then allows identification of those regions of the flow responsible for slow convergence and divergence respectively. Each eigenvector has a similar structure to a solution vector, with five complex components at each grid point; in Figure 3 the $L^{2}$ norm at each point is taken, representing the overall size of the vector in all components at that point. The two eigenvectors shown correspond to the eigenvalues marked a and $\mathrm{b}$ in the previous figure, and it should be noted that they therefore show different things: for Case 9 parts of the field that are most slowly damped, for Case 10 parts of the field that are diverging.

From these eigenmodes much information may be gleaned that is ordinarily unavailable. For example it is becomes clear that in Case 9 the convergence rate of the iteration is limited by the convergence of the flow in the regions of the shock and upper surface negative pressure gradient. Devising an FPI that improves the convergence somewhere else, e.g. near the stagnation point, would result in no overall improvement. Similarly in Case 10 the cause of the non-linear limit cycle instability has been positively identified as the separation, in particular the largest recirculating region immediately behind the shock. Any treatment of the instability, in either the non-linear or linear problem, must necessarily involve this region. In both modes dark spots appear in the field under the aerofoil where no special physical features are present, indicating mesh or discretization problems.

Of course the eigenmodes depend on the FPI, and so the analysis relates to physical phenomena only over the discretization. On the other hand this is a feature that makes the analysis useful in the study of FPIs. Further work will apply eigenmode analysis to attempt to systematically categorize the behaviour of some common FPIs with respect to certain flow features, the goal being to quantify the local influence of, for example different directional multigrid coarsening algorithms. The wider use of Krylov methods also introduces the need for an FPI that is a good Krylov preconditioner, though not necessary a good multigrid smoother. Where previously Fourier analysis 
was an essential tool for studying multigrid smoothers, we expect eigenmode analysis to be useful for studying Krylov preconditioners.

\section{Gradient-Based Optimization}

The adjoint method is now applied to the drag minimization of the DLR-F6 wing-body configuration at Mach 0.75 , a Reynolds number of $3 \times 10^{6}$, and $C_{L}=0.8$, at which conditions the case has a large region of separated flow in the junction between the upper surface of the wing and the fuselage, as well as along most of the length of the wing. For the adjoint problem on this geometry, adjointed LU-SGS with multigrid alone was unconditionally unstable, and applying RPM was necessary, see Figure 4. Also shown is the $\rho$-residual restricted to $\mathcal{P}$ and $\mathcal{Q}$, and the dimension of $V$. The corresponding eigenvalues in the same figure show that in total 8 unstable modes were found.

The optimization algorithm used is conjugate-gradients (CG), as in [8], where the angle-of-attack is varied to constrain the lift. The surface of the computational grid is shown in Figure 4, and is coarse, but sufficient to resolve the separation mentioned. The 84 paired nodes of a free-form deformation bounding box are also shown, whose vertical positions, as well as 12 additional wing twist variables were used to parameterize the wing. The pairing of nodes constrained the wing thickness. With such a large number of design variables only gradient-based optimization is viable, and only the adjoint method can deliver the gradient efficiently. Note that since the bounding box passes inside the fuselage, the wing-body junction also varies, and this is accounted for by the geometry and grid generation process. The metric sensitivities needed in the gradient calculation are evaluated by finite-differences on the mesh deformation.

The convergence of the optimization is shown in Figure 5, the horizontal axis shows the number of calls to the flow solver (both linear and non-linear), thereby approximately representing computational effort. Symbols indicate gradient evaluations. After 32 solver calls CG was unable to reduce the drag further, giving a final reduction of about 10 drag counts. In contrast a similar optimization with 42 parameters produced a reduction of only 8 counts on this mesh (in a similar CPU time) [9], emphasizing the need for a comprehensive parameterization. The optimization reduced the region of corner separation considerably, Figure 5, while not completely eliminating it, which is unlikely to be possible within the design space considered, as it does not allow deformation of the body.

\section{Conclusions}

The influence of separation on the convergence of the non-linear and the adjoint problem has been examined with the use of eigenmode analysis and the recursive projection method. This method was shown to stabilize the linear 
calculation in situations where the use of the exact adjointed FPI for the nonlinear problem was unstable. The resulting adjoint code was applied to the optimization of a wing-body configuration, whereby the region of separation was considerably reduced.
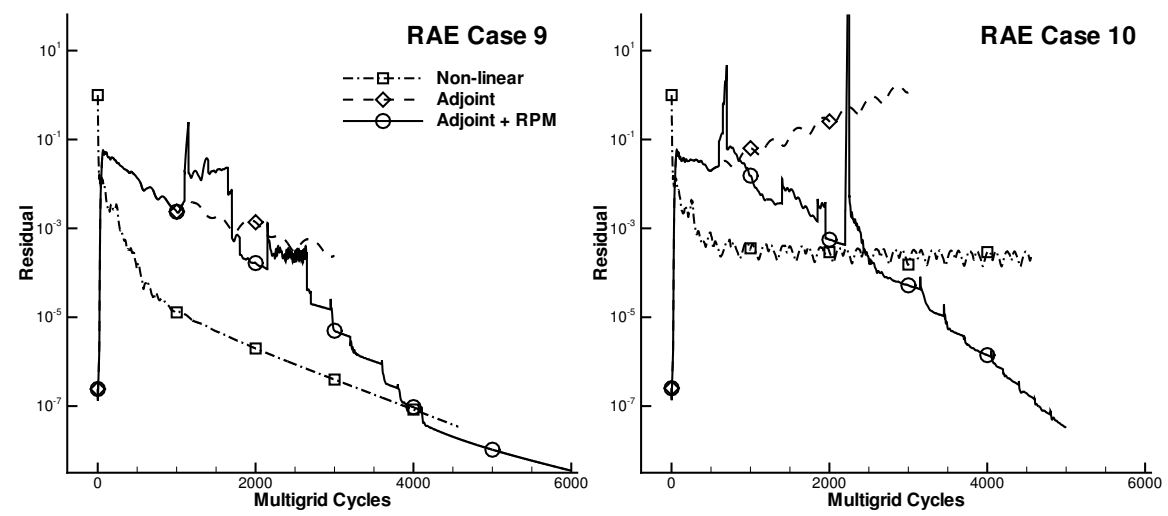

Figure 1 Non-linear and linear convergence histories for the two cases.
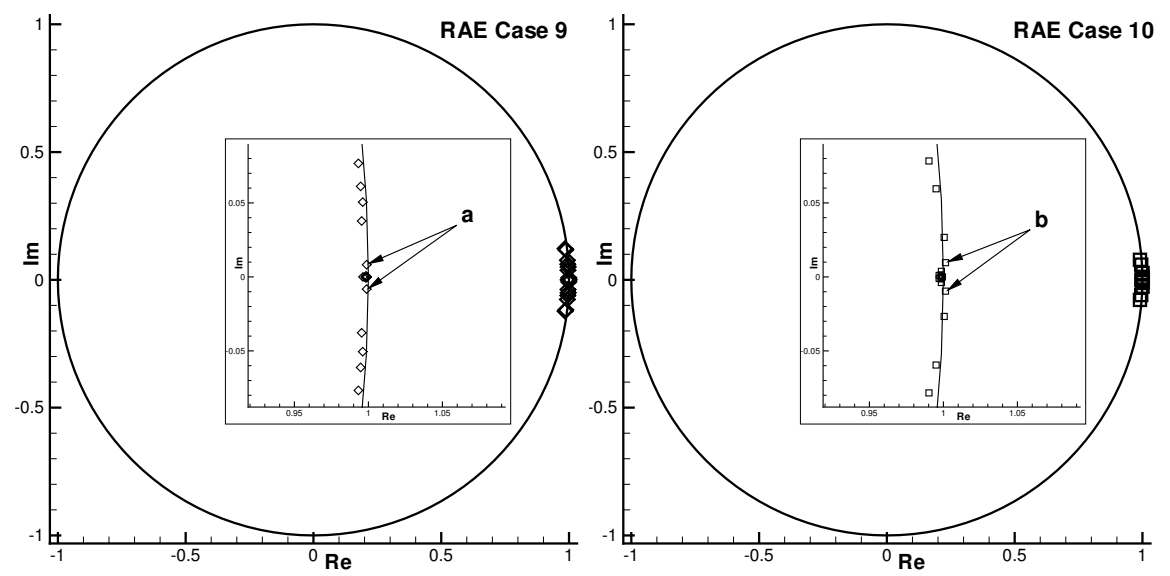

Figure 2 Dominant eigenvalues of the linear iterations on the two cases. 


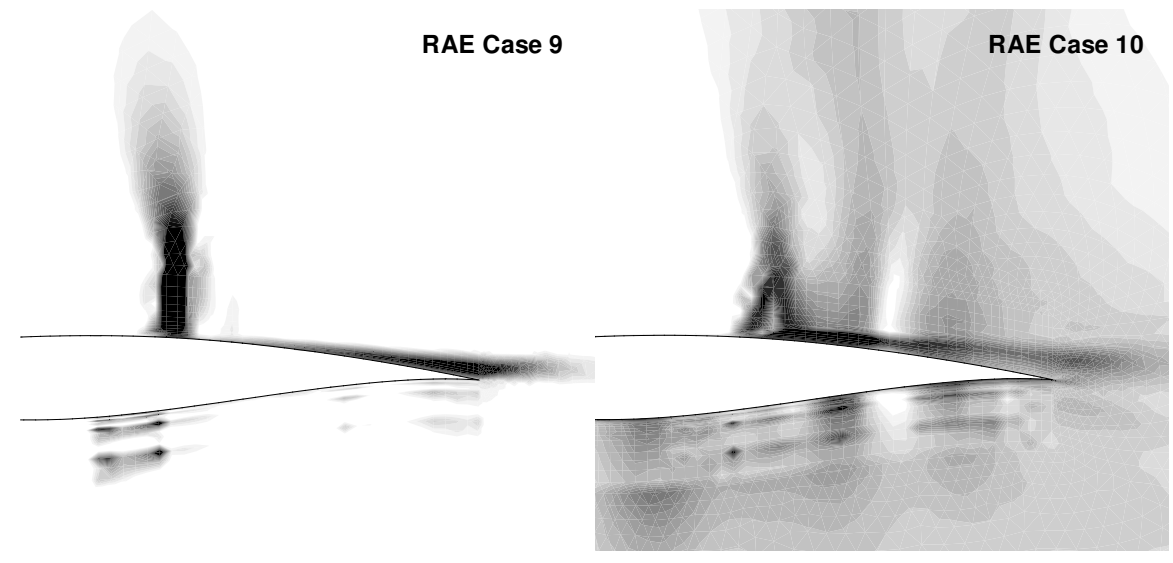

Figure 3 The dominant eigenvectors of each of the two cases; dark regions indicate large values. The corresponding eigenvalues are highlighted in Figure 2.
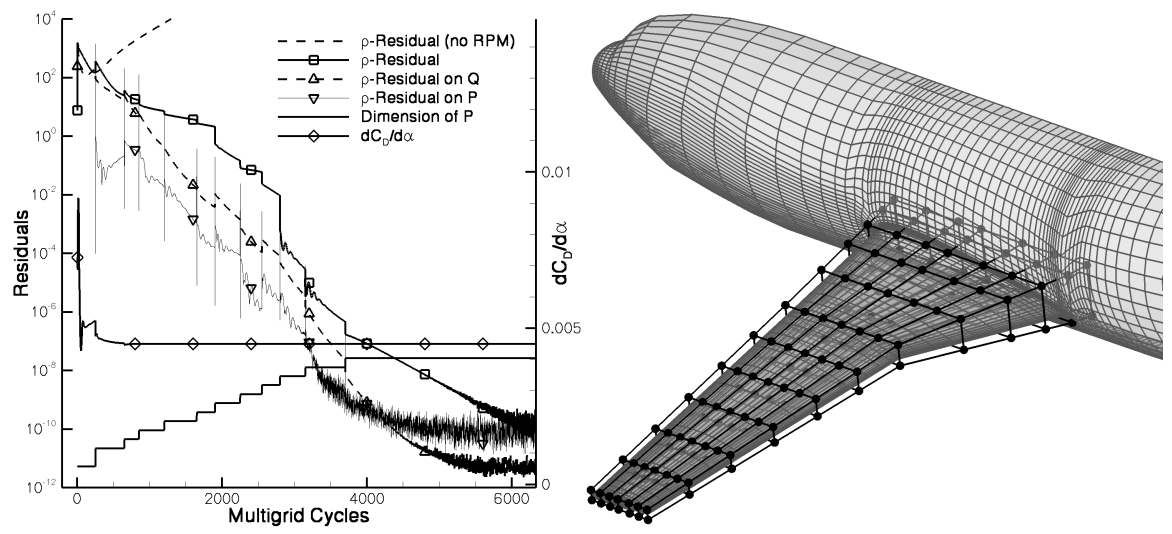

Figure 4 Stabilization of DLR-F6 adjoint computation with RPM, and parameterization of the wing with a free-form deformation box with 84 paired nodes. Twist is parameterized separately with 12 variables. 

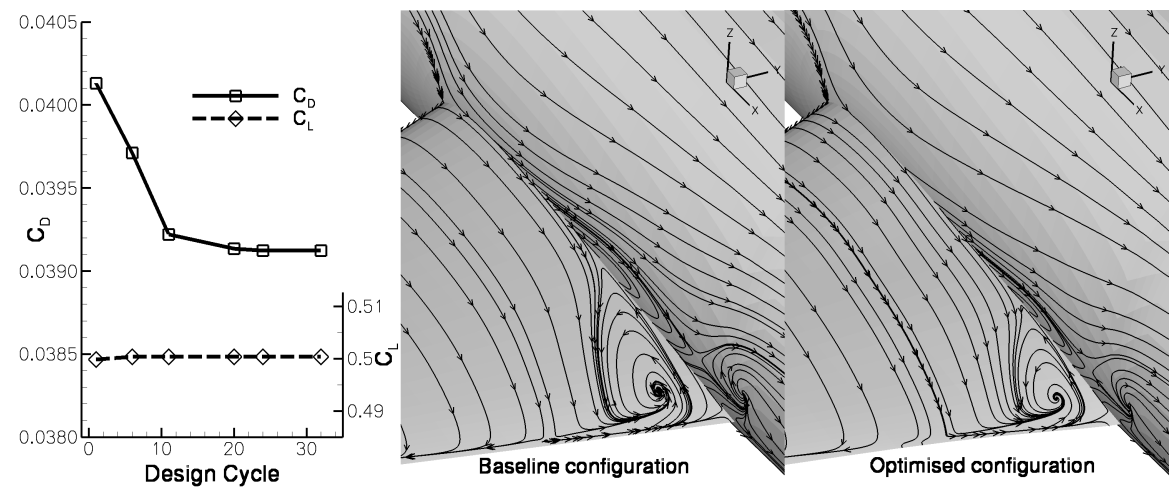

Figure 5 Convergence of the F6 drag-minimization optimization. Comparison of the region of corner separation before and after optimization.

\section{References}

[1] Dwight, R., Brezillon, J., Vollmer, D.: Efficient algorithms for solution of the adjoint compressible Navier-Stokes equations with applications. In: Proceedings of the ONERA-DLR Aerospace Symposium (ODAS), Toulouse. (2006)

[2] Gerhold, T., Galle, M., Friedrich, O., Evans, J.: Calculation of complex 3D configurations employing the DLR TAU-Code. In: American Institute of Aeronautics and Astronautics, Paper AIAA-97-0167. (1997)

[3] Dwight, R.: Efficiency Improvements of RANS-Based Analysis and Optimization using Implicit and Adjoint Methods on Unstructured Grids. PhD thesis, School of Mathematics, University of Manchester (2006)

[4] Schroff, G., Keller, H.: Stabilization of unstable procedures: The Recursive Projection Method. SIAM Journal of Numerical Analysis 30 (1993) 1099-1120

[5] Görtz, S., Möller, J.: Evaluation of the Recursive Projection Method for efficient unsteady turbulent CFD simulation. ICAS 2004 (2004)

[6] Campobasso, M., Giles, M.: Stabilization of a linear flow solver for turbomachinery aeroelasticity by means of the recursive projection method. AIAA Journal 42 (2004) 1765-1774

[7] Cook, P., McDonald, M., Firmin, M.: 6. Number 138 in AGARD-AR. In: Aerofoil RAE 2822 - Pressure Distributions and Boundary Layer and Wake Measurements. AGARD - Advisory Group for Aerospace Research \& Development, Neuilly-sur-Seine, France (1979)

[8] Dwight, R., Brezillon, J.: Effect of various approximations of the discrete adjoint on gradient-based optimization. In: Proceedings of the 44th AIAA Aerospace Sciences Meeting and Exhibit, Reno NV, AIAA-2006-0690. (2006)

[9] Brezillon, J., Brodersen, O., Dwight, R., Ronzheimer, A., Wild, J.: Development and application of a flexible and efficient environment for aerodynamic shape optimisation. In: Proceedings of the ONERA-DLR Aerospace Symposium (ODAS), Toulouse. (2006) 